\title{
Detection of sub-shot-noise spatial correlation in high-gain parametric down-conversion
}

\author{
O. Jedrkiewicz ${ }^{1}$, Y.-K Jiang ${ }^{2}$, E. Brambilla ${ }^{1}$, A. Gatti ${ }^{1}$, M. Bache ${ }^{1}$, L. A. Lugiato ${ }^{1}$, and P. Di Trapani ${ }^{1}$ \\ ${ }^{1}$ INFM, Dipartimento di Fisica e Matematica, Universita' dell'Insubria, Via Valleggio 11, 22100 Como, Italy \\ ${ }^{2}$ Department of Electronic Science and Applied Physics, Fuzhou University, 350002 Fuzhou, China
}

(Dated: October 10, 2018)

\begin{abstract}
Using a 1GW-1ps pump laser pulse in high gain parametric down-conversion allows us to detect sub-shot-noise spatial quantum correlation with up to one hundred photoelectrons per mode, by means of a high efficiency CCD. The statistics is performed in single-shot over independent spatial replica of the system. The paper highlights the evidence of quantum correlation between symmetrical signal and idler spatial areas in the far field, in the high gain regime. In accordance with the predictions of numerical calculations the observed transition from the quantum to the classical regime is interpreted as a consequence of the narrowing of the down-converted beams in the very high gain regime.
\end{abstract}

PACS numbers:

Spatial quantum optical fluctuations are studied because of new potential applications of quantum optical procedures in parallel processing and multi-channel operation. Examples are quantum holography [1], the quantum teleportation of optical images [2], and the measurements of small displacements beyond the Rayleigh limit [3]. There is now a large literature on spatial effects in the spontaneous regime of parametric down-conversion (PDC) where photons are created one pair at a time [4]. The process of PDC is in fact particularly suitable for the study of spatial correlations because of its large emission bandwidth in the spatial frequency domain [5]. Nevertheless to date most spatial correlation measurements in PDC have been performed in single photon counts regime 6, 7] without evidencing any relevant quantum effects. The quantum twin beam character of the PDC emission has been evidenced in [8] by using low-to-medium pumppower lasers $(\leq 1 \mathrm{MW})$ and relying on statistical ensembles from different temporal replicas of the system. With increasing gain a transition from the quantum to the classical regime has been observed [9]. However, recent theoretical investigations predict multi-mode spatial quantum correlations (sub-shot-noise photon-number correlation between symmetrical portions of the signal and idler angular distributions) also for arbitrarily high gains, provided that the detection area exceeds the typical size of the mode (coherence area) 10, 11].

Here we report on the first quantum spatial measurements of PDC radiation performed by using a lowrepetition rate $(2 \mathrm{~Hz})$ pulsed high-power laser $(1 \mathrm{GW}$ $1 \mathrm{ps})$. This enables us to tune the PDC to the high-gain regime while keeping a large pump beam size $(\sim 1 \mathrm{~mm})$. The huge number of transverse modes (roughly given by the ratio between: (i) the area of the near-field gain profile and (ii) the inverse of the angular bandwidth of the PDC process) allows us to identify regions of the signal and idler beams where symmetrical signal-idler pixel pairs correspond to independent spatial replica of the quantum system. We concentrate on a portion of the parametric fluorescence emitted close to the collinear direction and within a narrow frequency bandwidth around degeneracy. The generated pairs of signal and idler phase-conjugate modes propagate at symmetrical angles with respect to the pump direction in order to fulfil the phase-matching constraints, and each pair of symmetrical spots characterizing the far field represents a spatial replica. Thanks to the very large number of these, the statistical ensemble averaging necessary for the quantum measurement can be solely done over the spatial replicas for each, single, pump-laser pulse. A characterization of the system over several shots was only made in our case in order to verify that the selected spatial replicas are indeed statistically identical, as required for the suitable definition of the ensemble. The single-shot measurements reveal sub-shot-noise spatial correlations for a PDC gain $G$ (intensity amplification factor) leading to the detection of up to $\simeq 100$ photoelectrons per mode. Finally, by numerically solving the three-waves coupled equations in the framework of a $3 \mathrm{D}+1$ quantum model, we are able to attribute the observed transition from quantum to classical regime to the near-field gain narrowing that occurs in presence of a bell-shaped pump beam, at very-high gain.

The experimental setup is sketched in Fig. 1 The third harmonic $(352 \mathrm{~nm})$ of a 1ps, chirped-pulse amplified Nd:glass laser (TWINKLE, Light Conversion Ltd.) is used to pump a type II $5 \times 7 \times 4 \mathrm{~mm}^{3} \beta$-barium borate (BBO) non-linear crystal, operated in the regime of parametric amplification of the vacuum-state fluctuations. The input and output facets of the crystal are antireflection coated at $352 \mathrm{~nm}$ and $704 \mathrm{~nm}$, respectively. The pump beam is spatially filtered and collimated to a beam waist of approximately $1 \mathrm{~mm}$ (FWHM) at the crystal input facet. The energy of the $352 \mathrm{~nm}$ pump pulse can be continuously tuned in the range 0.1-0.4 mJ by means of suitable attenuating filters and by changing the energy of the $1055 \mathrm{~nm}$ pump laser pulse, allowing to have 
a gain in the range $10 \leq G \leq 10^{3}$. The parametric fluorescence at the horizontally polarized signal and vertically polarized idler modes is emitted over two cones, whose apertures depend on the specific wavelengths (see, e.g., 13, 14]). The BBO crystal $\left(\theta=49.05^{\circ}, \phi=0\right)$ is oriented in order to generate signal and idler radiation cones tangent in the collinear direction at the degenerate wavelength $\omega_{s}=\omega_{i}=\omega_{p} / 2(s, i$ and $p$ referring to signal, idler and pump respectively). The fluorescence around the collinear direction is selected by means of a $5 \mathrm{~mm} \times 8 \mathrm{~mm}$ aperture, placed $15 \mathrm{~cm}$ from the output facet of the BBO. The aperture turned out to be necessary in order to prevent beam clipping by the PBS, otherwise giving rise to substantial scattered radiation. The selected portion of the beam is transmitted through a polarizing beam splitter (PBS), which separates the signal and idler beams. The latter are finally sent onto two separate regions of a deep-depletion back illuminated charged coupled device (CCD) camera 15] (Roper Scientific NTE/CCD-400EHRBG1, 16 bits dynamical range, quantum efficiency $\eta \approx 89 \%$ at $704 \mathrm{~nm}$ at $-40^{\circ} \mathrm{C}$, dark current and read out noise $<1 e / \mathrm{pixel} / \mathrm{s}$ and $<3 e / \mathrm{pixel} / \mathrm{s}$ respectively), placed in the common focal plane of the two lenses $(f=10 \mathrm{~cm})$ used to image the signal and idler far fields. The detection array has 1340x400 pixels, with a pixel size of $20 \mu \mathrm{mx} 20 \mu \mathrm{m}$. Prior to the experiment the CCD was calibrated with a coherent source allowing the retrieval of spatial shot-noise statistics in its full dynamic range [16]. In our setup the correlation measurements are performed without using any narrow-band interferential filters (IFs), in contrast to the case of photon-counting experiments (coincidence measurements), since IFs unavoidably introduce relevant transmission losses reducing the visibility of sub-shot noise correlations. The pumpfrequency contribution is removed by using normal incidence $\left(M_{5}\right)$ and at $45 \mathrm{deg}\left(M_{4}\right)$ high-reflectivity (HR) mirrors coated for $352 \mathrm{~nm}$ placed before and after the PBS, respectively, and a low-band pass color filter $(90 \%$ transmission around $704 \mathrm{~nm}$ ) placed in front of the CCD. A further HR@352nm mirror $\left(M_{4}^{\prime}\right)$ is placed in one of the two arms at a suitable angle in order to balance the unequal transmission of the PBS in the two arms. All the optical components (except the color filter) have antireflection coatings at $704 \mathrm{~nm}$. The estimated quantum efficiency of each detection line, which accounts for both the transmission losses and the detector efficiency, is $\eta_{t o t} \simeq 75 \%$.

Fig. 22 shows a typical far field image recorded in a single shot, where a fairly broadband radiation (i.e. the one transmitted by the rectangular aperture) is acquired in the signal (left) and idler (right) branches. The selection of the desired temporal and angular bandwidth around degeneracy is made by temporarily inserting in front of the CCD a 10nm wide IF around $704 \mathrm{~nm}$, allowing us to locate the collinear degeneracy point (see Fig. 2]). The data analysis is limited within two rectangu-

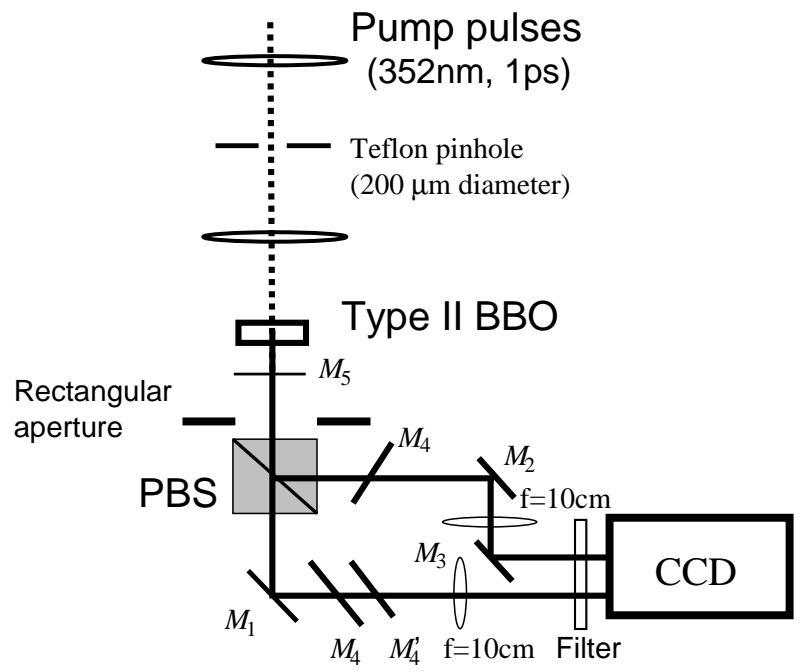

FIG. 1: Scheme of the experimental set-up used for the spatial correlation measurements (see text).

lar boxes (black frames in Fig 2a) corresponding to an angular bandwidth of $20 \mathrm{mrad} \times 8 \mathrm{mrad}$ and to a temporal bandwidth smaller than $10 \mathrm{~nm}$. The selected regions contain 4000 pixels each. In this work we investigate pixel pair correlation, and since the size of the CCD pixel approximately corresponds to the physical size of the replica, the ensemble is large enough to perform the desired statistics. We have observed that much larger boxes worsen the level of the correlation; this is attributed to residual scattering owing to diffraction from the borders of the aperture but also to the contribution of other frequency components (far from degeneracy) characterized by a lower angular symmetry between signal and idler cones. A zoom of the selected areas is presented in Fig 22, where the rather spectacular symmetry of the intensity distribution in the signal and idler branches shows the twin-beam character of the phase-conjugate modes.

The aim of the experiment is to quantify the symmetrical pixel pair correlation. This is done by measuring the variance $\sigma_{s-i}^{2}$ of the PDC photoelectronnumber difference $n_{s}-n_{i}$ of the signal/idler pixel pair versus the mean total number of down-converted photoelectrons (pe) of the pixel pair. This variance is $\sigma_{s-i}^{2}=\left\langle\left(n_{s}-n_{i}\right)^{2}\right\rangle-\left\langle n_{s}-n_{i}\right\rangle^{2}$ where the averages are spatial averages performed over all the symmetrical pixel pairs contained in the chosen regions. Each single shot of the laser provides a different ensemble, characterized by its pixel pair average pe number $\left\langle n_{s}+n_{i}\right\rangle$, in turn related to the parametric gain. In the experiment, ensembles corresponding to different gains are obtained by varying the pump-pulse energy. We note that the readout noise of the detector, its dark current, and some unavoidable light scattered from the pump, signal and idler fields contribute with a non-negligible background noise to the process. This is taken into account by applying a 
(a)

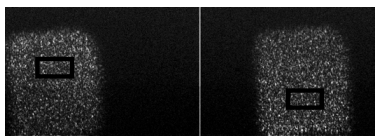

(b)

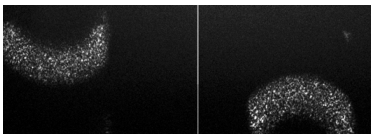

(c)

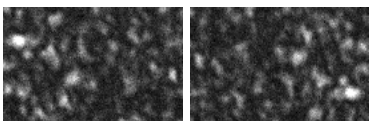

FIG. 2: (a) Single-shot far field image recorded by the CCD for a pump beam waist $w_{0} \simeq 1 \mathrm{~mm}$ and pump energy $\varepsilon_{p} \simeq$ $0.3 m J$. The spatial areas for statistics are delimited by the white boxes selected within the degenerate signal and idler modes, spatially localized from the single shot image recorded with the $10 \mathrm{~nm}$-broad IF (b). (c) Zoom of two symmetrical areas of the signal and idler far fields.

standard correction procedure (see for example $[18]$ ), by subtracting the background fluctuations $\sigma_{b}^{2}$ from the $e f$ fectively measured variance $\sigma_{(s+b)-(i+b)}^{2}$ of the total intensity difference (signal+background)-(idler+background) obtaining $\sigma_{s-i}^{2}=\sigma_{(s+b)-(i+b)}^{2}-2 \sigma_{b}^{2}$. This background noise, having a standard deviation of 7 counts $( \pm 0.1$ from shot to shot, estimated by repeating the measurement with the same pump-pulse energy) is measured in presence of pulse illumination over an area of the same size of the acquisition area and suitably displaced from the directly illuminated region. The validity of the data correction procedure is tested by sending in the setup (with no crystal) through the PBS a coherent circularly polarized pulsed beam (@704nm), and verifying for different laser energies that the intensity difference fluctuations from the two coherent portions of beams recorded on the CCD lie at the shot noise level.

Fig. 3 shows the experimental results where each point is associated with a different laser shot. The data are normalized to the shot noise level (SNL), and their statistical spread accounts for the background correction. Al-



FIG. 3: Intensity difference variance $\sigma_{s-i}^{2}$ normalized to the SNL $\left\langle n_{s}+n_{i}\right\rangle$. Each point (white circle) corresponds to a single shot measurement where the spatial ensemble statistics has been performed over a $100 \times 40$ pixels region. The triangles (each one obtained by averaging the experimental points corresponding to a certain gain) and their linear fit illustrate the trend of the data in the region between $\left\langle n_{s}+n_{i}\right\rangle$ $=8$ and 20. Inset: Typical correlation degree profile in the regime where $\left\langle n_{s}+n_{i}\right\rangle \simeq 8$ (see text).

though the noise on the individual signal and idler beams is found to be very high and much greater than the standard quantum limit $\left(=\left\langle n_{s}\right\rangle\right.$ and $\left\langle n_{i}\right\rangle$ respectively), we observe an evident sub-shot noise pixel pair correlation up to gains characterized by $\left\langle n_{s}+n_{i}\right\rangle \approx 15-18$. Since in that regime the observed transverse size of the coherence areas (i.e. of the modes) is about 2-4 pixels, this approximately corresponds to 100 pe per mode. We can have an idea of the transverse size of the mode by looking at the standard two-dimensional cross-correlation degree $\gamma=\left(\left\langle n_{s} n_{i}\right\rangle-\left\langle n_{s}\right\rangle\left\langle n_{i}\right\rangle\right) / \sqrt{\sigma_{s}^{2} \sigma_{i}^{2}}$, between all the angularly symmetrical signal and idler pixels contained within the black boxes (see Fig. 2a). This can be plotted for instance as a function of the horizontal and vertical shifts of the recorded image on the CCD, keeping fixed the position of the boxes. In general $|\gamma| \leq 1$ with $\gamma=1$ for perfect correlation. A transverse section of the correlation function obtained from a single-shot image characterized by $\left\langle n_{s}+n_{i}\right\rangle \approx 8$ is plotted in the inset of Fig. 3 as a function of the horizontal shift $x$ (in pixels units). As expected, virtually perfect correlation (in our case the peak value is $\simeq 0.99$ ) is obtained for perfect determination (i.e. within one pixel) of the center of symmetry between the signal and the idler regions.

In order to interpret the observed transition from quantum to classical regime we present in Fig. 目 the results of the numerical calculations. The full quantum model accounts for the two transverse and the temporal degrees of freedom with propagation along the crystal, for the 


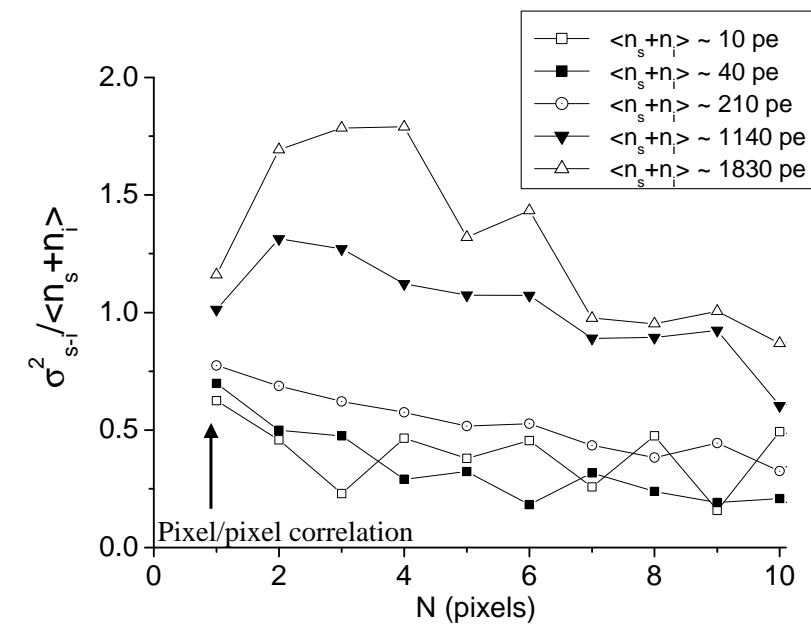

FIG. 4: Numerical calculation of $\sigma_{s-i}^{2}$ (normalized to SNL) between symmetrical portions of signal and idler plotted as a function of the detection area represented by $\mathrm{N} \times \mathrm{N}$ binned pixels. Different curves correspond to different values of the gain characterized by the mean number of down-converted pe per pixel pair $\left\langle n_{s}+n_{i}\right\rangle$.

angular and chromatic material dispersion up to the second order, and for the finite spatial and temporal widths of the Gaussian pump pulse. The crystal and inputpulse parameters are those relative to the experiment. The figure presents $\sigma_{s-i}^{2}$, normalized to the SNL, vs the size of the detection area for different gains. Each point is the result of a statistics performed over one single laser-shot. The case $\mathrm{N}=1$ corresponds to the experiment. The simulations (data not shown) outline that, in spite of the fixed pump-beam diameter, the signal and idler beam diameters at the crystal output strongly depend on the gain and decrease when the latter increases. This can be easily interpreted when considering that the signal and idler beam size maps not the pump-beam profile but the actual parametric amplification gain profile $G(\mathbf{r}) \sim \cosh ^{2}[\sigma \mathrm{A}(\mathbf{r}) \mathrm{L}][19]$ ( $L$ being the crystal length, $A$ the pump field amplitude and $\sigma$ a parameter proportional to the setting characteristics), as long as filtering due to the limited spatial bandwidth does not take place [20]. On narrowing the size of the PDC beams, the coherence areas in the far field (i.e. the modes) increase their size, as straightforward consequence of the convolution theorem in Fourier analysis 13. Since revealing quantum correlations requires detection areas larger (or comparable) to the mode size (as also discussed in [10]), it is necessary when increasing the gain to have larger detectors in order to obtain below-shot-noise variance as shown in Fig. 4 Note that Fig. 4 evidences the transition from quantum to classical regime in case of single-pixel detection $(\mathrm{N}=1)$ for a gain that is higher than in the experiment. Instead, in the experiment, excess noise is observed for $\left\langle n_{s}+n_{i}\right\rangle>20$, which we attribute first to the effect of residual scattered light whose contribution grows linearly with the radiation fluence and is thus expected to overcome the shot noise at large pumping, and second to the uncertainty in the determination of the symmetry center of the signal and idler image portions. In fact simulations have shown that an uncertainty as small as a few microns (i.e. a fraction of the pixel size, unavoidable experimentally), prevents to observe sub-shot-noise correlation as soon as $\left\langle n_{s}+n_{i}\right\rangle$ exceeds some tens of pe, while still preserving sub-shot-noise correlation for smaller gain values. Finally, the maximum level of noise reduction observed experimentally agrees with the theoretical limit (dotted line in Fig. (3) determined by the total losses of the system $\left(\sim 1-\eta_{t o t}[10]\right)$, in accordance with the result of Fig. [4

In conclusion, we have shown that twin beams of light generated in parametric down-conversion exhibit subshot noise spatial correlation by measuring an evident quantum noise reduction on the signal/idler intensity difference. A transition to above shot-noise correlation is observed as the gain increases. This quantum-to-classical transition, in agreement with numerical simulations, is explained as a narrowing of the signal/idler beams with increased gain. This leads in turn to a larger mode size and therefore also to the need of larger pixels to observe below shot-noise correlation [10]. This will be the aim of a future work. To our knowledge, this is the first experimental investigation of quantum spatial correlations in the high gain regime, where the huge number of transverse spatial modes is detected in single shot by means of a high-quantum-efficiency CCD.

This work has been supported by the European Union (QUANTIM contract IST-2000-26019). M. B. acknowledges support from the Carlsberg foundation.

[1] A. F. Abouraddy, B. E. A. Saleh, A. V. Sergienko, and M. C. Teich, Opt. Express 9, 498 (2001).

[2] I. V. Sokolov, M. I. Kolobov, A. Gatti, and L. A. Lugiato, Opt. Commun. 193, 175 (2001).

[3] N. Treps, U. Andersen, B. Buchler, P. K. Lam, A. Maitre, H.-A Bachor, and C. Fabre, Phys. Rev. Lett. 88, 203601 (2002).

[4] L. A. Lugiato, A. Gatti, and E. Brambilla, J. Opt. B: Quantum Semiclassical Opt. 4, S176 (2002), and references therein.

[5] F. Devaux and E. Lantz, Eur. Phys. J. D 8, 117 (2000).

[6] B. M. Jost, A. V. Sergienko, A. F. Abouraddy, B. E. A. Saleh, and M. C. Teich, Opt. Express 81, 3 (1998).

[7] S. S. R. Oemrawsingh, W. J. van Drunen, E. R. Eliel, and J. P. Woerdman, J. Opt. Soc. Am. B 19, 2391 (2002).

[8] O. Aytur and P. Kumar, Phys. Rev. Lett. 65, 1551 (1990).

[9] M. L. Marable, S.-K. Choi, and P. Kumar, Opt. Express 2, 84 (1998).

[10] E. Brambilla, A. Gatti, M. Bache, and L. A. Lugiato, Phys. Rev. A 69, 023802 (2004).

[11] A. Gatti, E. Brambilla, and L. A. Lugiato, Phys. Rev. Lett. 83, 1763 (1999). 
[12] A. Yariv, Quantum electronics (John Wiley and Sons, New York, 1989).

[13] A. Berzanskis, W. Chinaglia, L. A. Lugiato, K.-H. Feller, and P. Di Trapani, Phys. Rev. A 60, 1626 (1999).

[14] M. H. Rubin, Phys. Rev. A 54, 5349 (1996).

[15] J.R. Janesick, Scientific Charge-Coupled Devices (SPIE Press Bellingham, Washington, 2001), pp. 204-205; see also http://www.roperscientific.de/theory.html

[16] Y.-K. Jiang, O. Jedrkiewicz, S. Minardi, P. Di Trapani, A. Mosset, E. Lantz, and F. Devaux, Eur. Phys. J. D 22, 521 (2003).
[17] K. Koch, E. Cheung, G. T. Moore, S. H. Chakmakjian, and J. M. Liu, IEEE J. of quant. electron. 31, 769 (1995).

[18] A. Mosset, F. Devaux, G. Fanjoux, and E. Lantz, Eur. Phys. J. D 28, 447 (2004).

[19] S. A. Akhmanov, V. A. Vysloukh, A. S. Chirkin, Optics of Femtosecond Laser Pulses (American Institute of Physics, New York, 1992), p.151.

[20] P. Di Trapani, G. Valiulis, W. Chinaglia and A. Andreoni, Phys. Rev. Lett. 80, 265 (1998). 